\title{
Penetrative convection in a superposed porous-medium-fluid layer via internal heating
}

\author{
By MAGDA CARR \\ Division of Civil Engineering, University of Dundee, Dundee DD1 4HN, UK \\ m.carr@dundee.ac.uk
}

(Received 3 August 2003 and in revised form 1 March 2004)

Penetrative convection in a two-layer system in which a layer of fluid overlies and saturates a porous medium is simulated via internal heating. The motion in the porous medium is described via Darcy's law and in the fluid layer by the NavierStokes equations with a Boussinesq approximation. The lower porous surface is held fixed at a temperature $T_{L}$, while the upper fluid surface is stress free and held at $T_{U}>T_{L}$. Internal heating takes place in both layers and allows the model to describe penetrative convection. The strength of heating has a dramatic effect on both the onset of convection and the nature of the ensuing convection cells. It is found that a heat source/sink $Q$ in the fluid layer has a destabilizing effect on the porous layer whereas one in the porous medium $Q_{m}$ has a stabilizing influence on the fluid. The effect of $Q$ and $Q_{m}$ on their respective layers, however, depends strongly upon the temperature difference $T_{U}-T_{L}$, and the strength and type of heating in the opposite layer. When $Q$ and $Q_{m}$ are varied, a range of streamlines are presented that exhibit novel behaviour. The model is compared with an alternative in which the density is assumed to have quadratic temperature dependence and there is no internal heating. When the two models are mathematically adjoint they are shown to yield the same critical instability threshold but different eigenfunctions. It is also shown that the initiating cell is not necessarily the strongest one. This curious behaviour is explained and illustrated with a range of streamlines for variable permeability.

\section{Introduction}

The purpose of this paper is to provide a model, via internal heating, for penetrative convection when a fluid overlies and saturates a layer of porous material. Penetrative convection refers to convective motion which begins in an unstable layer, and penetrates into an otherwise stable layer or layers. There are several ways to describe penetrative convection, at least five of which are discussed in detail in Straughan (1993). One of the most widely employed models is internal heating. An internal heat source (or sink) can give rise to a situation where one part of a layer is naturally convecting while the other remains stable; hence penetrative convection can occur. Many references can be found in which convection via internal heating is described. One of the most significant contributions, from which great advancement has been made, is that of Roberts (1967). Roberts (1967) models convection in a horizontal layer of fluid cooled from above, thermally insulated from below and heated uniformly by an internal source. Matthews (1988) adapts the work of Roberts (1967) to formulate a model for the onset of penetrative convection in a single layer of fluid. Penetrative convection in a single porous layer has similarly been modelled, 
see Straughan \& Walker (1996). However, there is very little literature on penetrative convection in the coupled porous-medium-fluid system despite it having numerous important applications.

For example, ice may be treated as a porous medium if it has sufficiently high permeability, see Eicken et al. (2002). Hence, if the porous layer takes the form of ice, and water is the fluid, there is scope to model a wide range of important geophysical problems. These include sea ice melting in the Arctic (Notz et al. 2003) and mixing in ice covered lakes (Matthews 1988). In addition, further applications include penetrative convection in the solidification of alloys (Worster 1992), patterned ground formation under water (Carr \& Straughan 2003), contaminant transport due to flow of water under the Earth's surface (Curran \& Allen 1990; Allen \& Khosravani 1992; ElHabel, Mendoza \& Bagtzogloum 2002), flow of oil in underground reservoirs (Allen 1984; Ewing 1996), bio remediation of contaminated ground (Suchomel, Chen \& Allen $1998 a, b$ ), and hydrothermal synthesis in the growth of crystalline materials (Chen, Prasad \& Chatterjee 1999).

In this paper, penetrative convection in the coupled porous-medium-fluid system is simulated by internal heating in both layers. We believe this is the first time such a system has been modelled in this way. Standard convection was dealt with primarily by Nield (1977), with an important extension in Nield (1983). Chen \& Chen (1988, 1989) also analysed convection in the superposed porous-medium-fluid problem. They made the significant discovery that linear instability neutral curves may be bimodal, i.e. they may possess two local maxima. Chen \& Chen $(1988,1989)$ showed both analytically and experimentally that the local maxima correspond to a parameter range in which convection is initiated in either the fluid layer or the porous medium. Worster (1992) also found two modes when investigating compositional convection in the solidification of binary alloys. In this paper, numerical findings are presented that exhibit a similar bimodal behaviour to those of Worster (1992), yet are generated by a completely different physical process.

Heat sinks in each layer are dealt with primarily, but the effects of having a source in both layers or a source in one layer and a sink in the other are also considered. A vast array of steady-state solutions can be found and the more interesting of these are discussed in detail. In particular, two steady-state solutions are presented in which a stably stratified layer is bounded above and below by unstable layers and an unstably stratified layer is bounded by stable ones. It is found that both a heat source and sink in the fluid layer have a destabilizing effect on the porous medium, whereas heating in the porous medium has a stabilizing effect on the fluid layer. The effect of a source or sink on its own layer, however, is a lot more complicated. It depends strongly on both the strength of the source/sink in the other layer and the difference between $T_{U}$ and $T_{L}$. The instability of the two-layer system and the size of the ensuing convection cells are found to be very sensitive to change in the strength of the internal heating. A selection of streamlines are presented which exhibit novel behaviour when $Q$ and $Q_{m}$ are varied.

Carr \& Straughan (2003) also considered the porous-medium-fluid system but they employed a quadratic equation of state as opposed to internal heating to simulate penetrative convection. It is stressed that from both a mathematical and physical point of view, the model of Carr \& Straughan (2003) is very different to the one considered here, however. In some instances, however, the linearized forms of the two models are shown to be mathematically adjoint. When this is the case it is found that the two models yield the same critical instability threshold but different eigenfunctions. The physical quantities driving the motion are different 


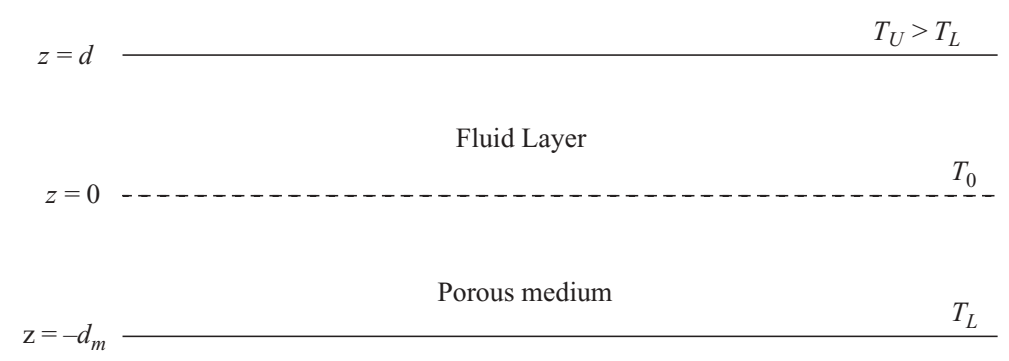

FIGURE 1. Schematic diagram of the governing system.

in the two systems and this is reflected in the pattern of the ensuing convection cells.

An outline of the paper is as follows. In the first two sections the governing system and corresponding equations are described and the non-dimensionalized perturbation equations derived. In $\S 4$ the boundary conditions are discussed and a linear instability analysis is given. A brief description of the numerical method is then provided in $\S 5$. An analytical and numerical discussion of the effect of including heat sinks in both layers on the stability of the system is presented in $\S 6$. This is extended to include all combinations of sources and sinks in $\S 7$. Finally in $\S 8$, a comparison is made between the model and that given in Carr \& Straughan (2003). An array of streamlines is presented showing that, contrary to intuition, the initiating cell is not necessarily the strongest one.

\section{The governing equations}

Consider a fluid occupying the three-dimensional layer $\left\{(x, y) \in \mathbb{R}^{2}\right\} \times\{z \in(0, d)\}$ saturating an underlying porous medium $\left\{(x, y) \in \mathbb{R}^{2}\right\} \times\left\{z \in\left(-d_{m}, 0\right)\right\}$. The interface between the saturated porous medium and the fluid is at $z=0$, see figure 1 . Suppose the density has a linear temperature dependence of the form

$$
\rho=\rho_{0}\left[1-\bar{\alpha}\left(T-T_{r}\right)\right],
$$

where $\rho, T$, and $\bar{\alpha}$ are density, temperature, and thermal expansion coefficient, and $\rho_{0}$ and $T_{r}$ are constant reference values for the density and temperature respectively. Then employing the Navier-Stokes equations, with a Boussinesq approximation, the governing equations for the fluid can be expressed as

$$
\begin{gathered}
\frac{\partial v_{i}}{\partial t}+v_{j} \frac{\partial v_{i}}{\partial x_{j}}=-\frac{1}{\rho_{0}} \frac{\partial p}{\partial x_{i}}+v \Delta v_{i}+\bar{\alpha} g T k_{i}, \\
\frac{\partial v_{i}}{\partial x_{i}}=0, \\
\frac{\partial T}{\partial t}+v_{i} \frac{\partial T}{\partial x_{i}}=\frac{k_{f}}{\left(\rho_{0} c_{p}\right)_{f}} \Delta T+2 Q,
\end{gathered}
$$

where (2.3) and (2.4) are the incompressibility condition and balance of energy, respectively. The $Q$ term in (2.4) is some (constant) internal heat source or sink and its inclusion allows the model to describe penetrative convection in the fluid layer (see §6). Equations (2.2)-(2.4) are assumed to hold for time $t>0$, in the spatial domain $\left\{(x, y) \in \mathbb{R}^{2}, z \in(0, d)\right\}$. The variables $v_{i}, t, x_{i}, p$, and $T$ are velocity, 
time, displacement, pressure and temperature, and $v, g, k_{f}, c_{p}$ are kinematic viscosity, gravity, thermal conductivity and specific heat at constant pressure. Standard indicial notation and the Einstein summation convention are employed throughout. Subscript (or superscript) $f$ or $m$ denotes fluid or porous medium, respectively. The symbol $\Delta$ is the Laplace operator and $\boldsymbol{k}=(0,0,1)$.

In the porous medium the motion of the fluid is described by Darcy flow with the buoyancy force again given by (2.1); therefore on $\left\{(x, y) \in \mathbb{R}^{2}\right\} \times\left\{z \in\left(-d_{m}, 0\right)\right\} \times$ $\{t>0\}$,

$$
\begin{gathered}
0=-\frac{1}{\rho_{0}} \frac{\partial p^{m}}{\partial x_{i}}-\frac{v}{K} v_{i}^{m}+\bar{\alpha} g T_{m} k_{i}, \\
\frac{\partial v_{i}^{m}}{\partial x_{i}}=0, \\
\frac{\left(\rho_{0} c_{p}\right)^{*}}{\left(\rho_{0} c_{p}\right)_{f}} \frac{\partial T_{m}}{\partial t}+v_{i}^{m} \frac{\partial T_{m}}{\partial x_{i}}=\frac{k^{*}}{\left(\rho_{0} c_{p}\right)_{f}} \Delta T_{m}+2 Q^{m},
\end{gathered}
$$

where $v_{i}^{m}, p_{m}, T_{m}$ are velocity, pressure and temperature in the porous medium. $K$ is the permeability and $Q^{m}$ is some (constant) internal heat source or sink in the porous layer. In (2.5) the acceleration term has been omitted since it is believed to be small and negligible (Nield \& Bejan 1999). Starred quantities are defined in terms of fluid and porous variables as $S^{*}=\phi S_{f}+(1-\phi) S_{m}$, where $\phi$ is the porosity and $S$ stands for a physical variable such as thermal conductivity $k$.

The temperatures on the upper and lower boundaries are held fixed at values $T_{U}$ and $T_{L}\left(<T_{U}\right)$ respectively. The governing equations (2.2)-(2.7) admit a steady-state solution in which the velocity field is zero and the unperturbed temperature profile is

$$
\begin{gathered}
\bar{T}(z)=-\frac{Q z^{2}}{\lambda}+\left(\frac{T_{U}-T_{0}}{d}+\frac{Q d}{\lambda}\right) z+T_{0}, \\
\bar{T}^{m}(z)=-\frac{Q_{m} z^{2}}{\lambda_{m}}+\left(\frac{T_{0}-T_{L}}{d_{m}}-\frac{Q_{m} d_{m}}{\lambda_{m}}\right) z+T_{0},
\end{gathered}
$$

where $\lambda=k_{f} /\left(\rho_{0} c_{p}\right)_{f}, \lambda_{m}=k^{*} /\left(\rho_{0} c_{p}\right)_{f}$ and $T_{0}$ is the temperature at the interface. Continuity of temperature and heat flux at $z=0$ yield the relation

$$
T_{0}=\mathscr{E}\left(T_{U}+\frac{Q d^{2}}{\lambda}+\frac{T_{L} \hat{d}}{\epsilon_{T}}+\frac{Q_{m} d_{m}^{2} \hat{d}}{\lambda_{m} \epsilon_{T}}\right),
$$

where $\mathscr{E}=\epsilon_{T} /\left(\epsilon_{T}+\hat{d}\right), \epsilon_{T}=\lambda / \lambda_{m}$ and $\hat{d}=d / d_{m}$.

\section{Non-dimensional perturbation equations}

To study the instability of the steady state, perturbations $\left(u_{i}, \theta, \pi, u_{i}^{m}, \theta_{m}, \pi_{m}\right)$ are introduced. Then non-dimensionalizing with fluid and porous-medium scalings of time, velocity, pressure, and temperature taken as

$$
\begin{aligned}
& \mathscr{T}=\frac{d^{2}}{v}, \quad U=\frac{v}{d}, \quad P=\frac{\rho_{0} \nu U}{d}, \quad T^{\#}=\frac{U}{\lambda} \sqrt{\frac{-Q \nu d}{g \bar{\alpha}}}, \\
& \mathscr{T}^{m}=\frac{d_{m}^{2}}{v}, \quad U^{m}=\frac{v}{d_{m}}, \quad P^{m}=\frac{\rho_{0} \nu U^{m}}{d_{m}}, \quad T^{m \#}=\frac{U_{m}}{\lambda_{m}} \sqrt{\frac{-Q_{m} \nu d_{m}^{3}}{g \bar{\alpha} K}},
\end{aligned}
$$


yields

$$
\begin{gathered}
\frac{\partial u_{i}}{\partial t}+u_{j} \frac{\partial u_{i}}{\partial x_{j}}=-\frac{\partial \pi}{\partial x_{i}}+\Delta u_{i}+R \theta k_{i}, \\
\frac{\partial u_{i}}{\partial x_{i}}=0, \\
\operatorname{Pr}\left(\frac{\partial \theta}{\partial t}+u_{i} \frac{\partial \theta}{\partial x_{i}}\right)=\Delta \theta+R f(z) w, \\
\delta^{2} \frac{\partial \pi^{m}}{\partial x_{i}}=-u_{i}^{m}+R^{m} \theta^{m} k_{i}, \\
\frac{\partial u_{i}^{m}}{\partial x_{i}}=0, \\
\operatorname{Pr}_{m}\left(G_{m} \frac{\partial \theta^{m}}{\partial t^{m}}+u_{i}^{m} \frac{\partial \theta^{m}}{\partial x_{i}}\right)=\Delta \theta^{m}+R^{m} f^{m}(z) w^{m},
\end{gathered}
$$

where $f(z)=1-2 z+\left(T_{U}-T_{0}\right) \lambda / Q d^{2}, w=u_{3}, G_{m}=\left(\rho_{0} c_{p}\right)^{*} /\left(\rho_{0} c_{p}\right)_{f}, f^{m}(z)=\left(T_{0}-\right.$ $\left.T_{L}\right) \lambda_{m} / Q_{m} d_{m}^{2}-2 z-1$ and $w^{m}=u_{3}^{m}$. The length scales in the fluid and porous domains are $d$ and $d_{m}$, respectively. In addition the fluid and porous-medium Rayleigh numbers $R a$ and $R a_{m}$ are defined as

$$
R a=R^{2}=\frac{-Q g \bar{\alpha} d^{5}}{\nu \lambda^{2}}, \quad R a_{m}=R_{m}^{2}=\frac{-Q_{m} g \bar{\alpha} K d_{m}^{3}}{\nu \lambda_{m}^{2}} .
$$

The Darcy number $\delta=\sqrt{K} / d_{m}$, and the fluid and porous-medium Prandtl numbers are $\operatorname{Pr}=v / \lambda, \operatorname{Pr}^{m}=v / \lambda_{m}$.

To ensure that $T^{\#}, T^{m \#}$ are real we impose $Q, Q_{m}<0$, i.e. we deal explicitly with a heat sink in both layers. It is worth noting that the non-dimensionalization is not unique. An alternative is to take the negative signs from $T^{\#}, T^{m \#}$ into the governing equations, then $Q, Q_{m}>0$ can be considered. This is discussed in more detail in $\S 7$.

\section{Boundary conditions and linearized instability theory}

Equations (3.1)-(3.6) are linearized and solutions sought of the form $u_{i}=u_{i}(\boldsymbol{x}) \mathrm{e}^{\sigma t}$. The time scale $t^{m}=t / \omega$ is introduced; then (3.1)-(3.6) yield

$$
\begin{gathered}
\sigma u_{i}=-\pi_{, i}+\Delta u_{i}+R \theta k_{i}, \\
u_{i, i}=0, \\
\sigma \operatorname{Pr} \theta=\Delta \theta+R f(z) w, \\
\delta^{2} \pi_{, i}^{m}=-u_{i}^{m}+R^{m} \theta^{m} k_{i}, \\
u_{i, i}^{m}=0, \\
\sigma \omega \operatorname{Pr}_{m} G_{m} \theta^{m}=\Delta \theta^{m}+R^{m} f^{m}(z) w^{m} .
\end{gathered}
$$

The upper surface is assumed to be open to the atmosphere (tangential stress free) and the lower boundary held fixed. Then, in non-dimensional form, the boundary conditions are

$$
\begin{aligned}
& \text { on } \quad z=1, \quad \theta=w=w_{, z z}=0 \\
& \text { on } \quad z=-1, \quad \theta^{m}=w^{m}=0 .
\end{aligned}
$$


At the interface, $z=0$, continuity of the normal components of velocity, temperature and heat flux, yields

$$
w^{m}=\frac{w}{\hat{d}}, \quad \theta^{m}=\frac{\delta}{\epsilon_{T}} \sqrt{\frac{\hat{A}}{\hat{d}}} \theta, \quad \frac{\partial \theta^{m}}{\partial z}=\delta \sqrt{\frac{\hat{A}}{\hat{d}^{3}}} \frac{\partial \theta}{\partial z},
$$

where $\hat{A}=Q / Q_{m}$. In addition at $z=0$, continuity of normal stress is required,

$$
\hat{d}^{2} \pi^{m}=\pi-2 w_{, z}
$$

and the Beavers-Joseph boundary condition is employed,

$$
\frac{\partial u^{\beta}}{\partial z}=\frac{\hat{d} \alpha}{\delta}\left(u^{\beta}-\hat{d} u_{m}^{\beta}\right), \quad \beta=1,2,
$$

where $\alpha$ is a coefficient depending on the fluid and porous medium under consideration. Further details of the last condition and alternatives are given in Nield \& Bejan (1999) and Straughan (2001).

A normal-mode representation is introduced of the form $w=W(z) g(x, y)$, $w^{m}=W^{m}(z) g^{m}(x, y)$, where $g(x, y)$ and $g^{m}(x, y)$ are horizontal plan forms satisfying $\Delta^{*} g=-a^{2} g, \Delta^{*} g^{m}=-a_{m}^{2} g^{m}, \Delta^{*}$ is the horizontal Laplacian and the wavenumber $a$ is a measure of the 'width' of the convection cell to the depth, $d$. The pressures $\pi$ and $\pi^{m}$ are eliminated by taking curlcurl of (4.1) and (4.4), and retaining the third component. Then regarding $A=\left(\mathrm{D}^{2}-a^{2}\right) W$ as an independent variable, where $\mathrm{D}^{2}=\mathrm{d}^{2} / \mathrm{d} z^{2}$, the governing equations yield five coupled second-order equations to determine the critical growth rate $\sigma$, namely,

$$
\begin{gathered}
\left(\mathrm{D}^{2}-a^{2}\right) W=A, \\
\left(\mathrm{D}^{2}-a^{2}\right) A-R a^{2} \Theta=\sigma A, \\
\left(\mathrm{D}^{2}-a^{2}\right) \Theta+R f(z) W=\sigma \operatorname{Pr} \Theta, \\
\left(\mathrm{D}^{2}-a_{m}^{2}\right) W^{m}+R^{m} a_{m}^{2} \Theta^{m}=0, \\
\left(\mathrm{D}^{2}-a_{m}^{2}\right) \Theta^{m}+R^{m} f^{m}(z) W^{m}=\sigma \omega P r_{m} G_{m} \Theta^{m},
\end{gathered}
$$

where (4.12)-(4.14) hold on $z \in(0,1)$, while (4.15)-(4.16) hold on $z \in(-1,0)$. Coupling is through the boundary conditions (4.7)-(4.11), which are reduced to yield

$$
\left.\begin{array}{c}
\Theta=W=A=0 \quad \text { on } \quad z=1, \\
\Theta^{m}=W^{m}=0 \quad \text { on } \quad z=-1, \\
W=\hat{d} W^{m}, \quad \Theta^{m}=\frac{\delta}{\epsilon_{T}} \sqrt{\frac{\hat{A}}{\hat{d}}} \Theta, \quad \mathrm{D} \Theta^{m}=\delta \sqrt{\frac{\hat{A}}{\hat{d}^{3}}} \mathrm{D} \Theta, \\
2 a^{2} \mathrm{D} W-\mathrm{D} A-\frac{\hat{d}^{2}}{\delta^{2}} \mathrm{D} W^{m}=-\sigma \mathrm{D} W, \\
a^{2} W+A=\frac{\hat{d} \alpha}{\delta}\left(\mathrm{D} W-\hat{d} \mathrm{D} W^{m}\right) \quad \text { on } \quad z=0 .
\end{array}\right\}
$$

Thus, our goal is to solve (4.12)-(4.16) subject to (4.17)-(4.19).

\section{The $\mathrm{D}^{2}-$ Chebyshev tau method}

To solve the eigenvalue problem (4.12)-(4.19), the $\mathrm{D}^{2}$-Chebyshev tau method was employed. This method is very accurate and allows as many eigenvalues as needed to be calculated. The corresponding eigenfunctions can also be easily computed. 
Before implementing the $\mathrm{D}^{2}$-Chebyshev tau method, (4.12)-(4.19) are transformed to the Chebyshev domain $(-1,1)$ via $\hat{z}=2 z-1$ and $\hat{z}_{m}=-2 z_{m}-1$. Then the fluid surface $z=1$ becomes $\hat{z}=1$ and the porous base $z_{m}=-1$ becomes $\hat{z}_{m}=1$ while the interface $z=0=z_{m}$ becomes $\hat{z}=-1=\hat{z}_{m}$.

The five quantities $W, A, \Theta, W_{m}$ and $\Theta_{m}$ are regarded as independent variables and expanded as Chebyshev series, e.g. $W=\sum_{n=0}^{N+2} W_{n} T_{n}(z)$. Equations (4.12)-(4.16), and the operator $\mathrm{D}^{2}$ are written in matrix form (Dongarra, Straughan $\&$ Walker 1996). The boundary conditions (4.17)-(4.19) are imposed by removing the $m(N+2)$ and $m(N+3)$ rows of the resulting matrix, $m=1, \ldots, 5$, and replacing them with the discrete forms of the boundary conditions. The boundary conditions are realized with the aid of the relations $T_{n}( \pm 1)=( \pm 1)^{n}$ and $T_{n}^{\prime}( \pm 1)=( \pm 1)^{n-1} n^{2}$. This results in a generalized $5(N+3) \times 5(N+3)$ matrix eigenvalue problem of the form $\boldsymbol{A} \boldsymbol{x}=\sigma \boldsymbol{B} \boldsymbol{x}$. This is solved for the eigenvalues $\sigma^{(n)}, n=1,2, \ldots$, and the eigenfunctions $\boldsymbol{x}^{(n)}$ with the aid of the QZ algorithm which was employed via the NAG routine F02BJF.

\section{Discussion of the model and numerical results}

Recall from $\S 2$, that the steady state-temperature profiles and the temperature at the interface are given by

$$
\begin{gathered}
\bar{T}(z)=-\frac{Q z^{2}}{\lambda}+\left(\frac{T_{U}-T_{0}}{d}+\frac{Q d}{\lambda}\right) z+T_{0}, \\
\bar{T}^{m}(z)=-\frac{Q_{m} z^{2}}{\lambda_{m}}+\left(\frac{T_{0}-T_{L}}{d_{m}}-\frac{Q_{m} d_{m}}{\lambda_{m}}\right) z+T_{0}, \\
T_{0}=\mathscr{E}\left(T_{U}+\frac{Q d^{2}}{\lambda}+\frac{T_{L} \hat{d}}{\epsilon_{T}}+\frac{Q_{m} d_{m}^{2} \hat{d}}{\lambda_{m} \epsilon_{T}}\right) .
\end{gathered}
$$

Differentiating (6.1) and (6.2), and eliminating $T_{0}$ yields the turning points for $\bar{T}$ and $\bar{T}^{m}$,

$$
\begin{aligned}
& z_{t p}=\frac{d(1-\mathscr{E})}{2}\left[\hat{\epsilon}\left(T_{U}-T_{L}-\frac{1}{\hat{\epsilon}_{m}}\right)+1\right], \\
& z_{t p}^{m}=\frac{d_{m} \mathscr{E}}{2}\left[\hat{\epsilon}_{m}\left(T_{U}-T_{L}+\frac{1}{\hat{\epsilon}}\right)-1\right],
\end{aligned}
$$

where $\hat{\epsilon}=\lambda / Q d^{2}$ and $\hat{\epsilon}_{m}=\lambda_{m} / Q_{m} d_{m}^{2}$. Since $Q, Q_{m}<0$ the turning points are both minimum points $\left(\bar{T}^{\prime \prime}, \bar{T}_{m}^{\prime \prime}>0\right)$. To investigate what type of steady-state temperature profile can be obtained, (6.3)-(6.5) are used to derive the identities

$$
\begin{aligned}
z_{t p}>0 & \Leftrightarrow T_{U}-T_{L}<-\frac{1}{\hat{\epsilon}}+\frac{1}{\hat{\epsilon}_{m}}, \\
z_{t p}<0 & \Leftrightarrow T_{U}-T_{L}>-\frac{1}{\hat{\epsilon}}+\frac{1}{\hat{\epsilon}_{m}}, \\
z_{t p}^{m}>-d_{m} & \Leftrightarrow T_{U}-T_{L}<-\frac{1}{\hat{\epsilon}}-\frac{1}{\hat{\epsilon}_{m}}\left(\frac{2 \hat{d}}{\epsilon_{T}}+1\right), \\
z_{t p}^{m}<-d_{m} & \Leftrightarrow T_{U}-T_{L}>-\frac{1}{\hat{\epsilon}}-\frac{1}{\hat{\epsilon}_{m}}\left(\frac{2 \hat{d}}{\epsilon_{T}}+1\right),
\end{aligned}
$$




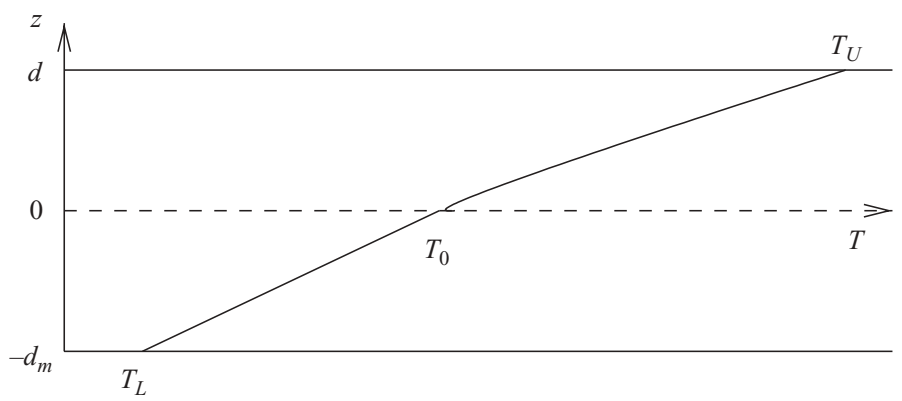

FIGURE 2. Steady-state temperature profile, case 1: depth $z$ against temperature $T$. $z_{t p}<0, z_{t p}^{m}<-d_{m}$.

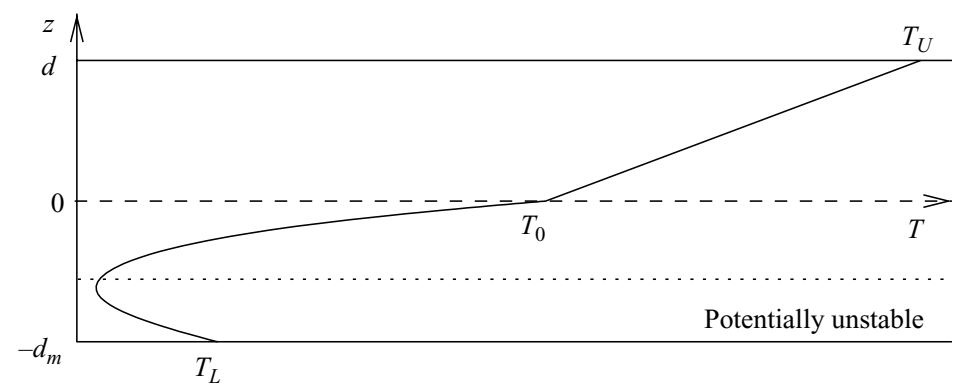

FIGURE 3. Steady-state temperature profile, case 2: depth $z$ against temperature $T$.

$$
\begin{gathered}
z_{t p}<0,-d_{m}<z_{t p}^{m}<0, T_{0}>T_{L} . \\
T_{0}>T_{L} \Leftrightarrow T_{U}-T_{L}>-\frac{1}{\hat{\epsilon}}-\frac{1}{\hat{\epsilon}_{m}} \frac{\hat{d}}{\epsilon_{T}}, \\
T_{0}<T_{L} \Leftrightarrow T_{U}-T_{L}<-\frac{1}{\hat{\epsilon}}-\frac{1}{\hat{\epsilon}_{m}} \frac{\hat{d}}{\epsilon_{T}} .
\end{gathered}
$$

Equations (6.6), (6.5) and (6.11) can be used to derive the relation

$$
z_{t p}>0 \quad \Leftrightarrow \quad z_{t p}^{m}>0 \quad \Rightarrow \quad T_{0}<T_{L} .
$$

Similarly, (6.7), (6.5) and (6.10) yield

$$
z_{t p}<0 \quad \Leftrightarrow \quad z_{t p}^{m}<0 \quad \Rightarrow \quad T_{0}>T_{L} .
$$

Hence, only the following four types of steady-state temperature profile are possible. It is worth noting at this point that the temperature difference between the interface and base is crucial in determining the stability characteristics. Thus, a distinction is made between cases when $T_{0} \lessgtr T_{L}$.

Case 1: The whole system is stable. If $z_{t p}^{m}<-d_{m}$, then (6.9) implies that $T_{0}>T_{L}$ and $z_{t p}<0$. Hence, a profile like that displayed in figure 2 is obtained. Recall that density is a linearly decreasing function of temperature (see (2.1)), i.e. the warmer the fluid the lighter it is. Thus, for the profile given in figure 2 the system is always stable.

Case 2: There is an unstable region in the porous layer and $T_{0}>T_{L}$. The profile given in figure 3 can be obtained by imposing (6.8) and (6.10) (note (6.7) follows automatically). 


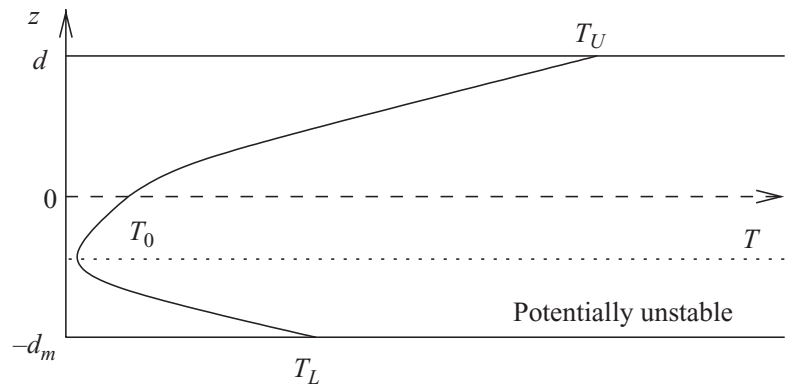

FIGURE 4. Steady-state temperature profile, case $3:$ depth $z$ against temperature $T$. $z_{t p}<0,-d_{m}<z_{t p}^{m}<0, T_{0}<T_{L}$.

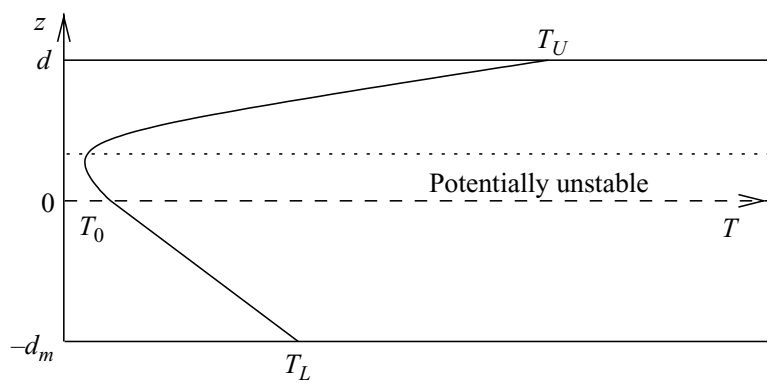

FIGURE 5. Steady-state temperature profile, case 4: depth $z$ against temperature $T$. $z_{t p}>0, z_{t p}^{m}>0, T_{0}<T_{L}$.

Fluid at the turning point, $z_{t p}^{m}$, is colder and heavier than at the base $z=-d_{m}$. Therefore, there is a potentially unstable region between the turning point and the base of the porous layer.

Case 3: There is an unstable region in the porous layer and $T_{0}<T_{L}$. The profile given in figure 4 can be obtained by imposing (6.7) and (6.11) (note (6.8) follows automatically). Again, there is a potentially unstable layer between the turning point and the base.

Case 4: There is an unstable region in the fluid and underlying porous layer, and $T_{0}<T_{L}$. If $z_{t p}>0$ then (6.6) implies that $T_{0}<T_{L}$ and $z_{t p}^{m}>0$. Thus, a profile like that displayed in figure 5 is obtained. There is a potentially unstable layer in the lower fluid region and underlying porous medium (everywhere beneath the dotted line).

Further steady-state profiles can be obtained for $Q, Q_{m}>0$ or $Q$ and $Q_{m}$ of alternating signs in an entirely analogous fashion to that presented above. Two specific cases in which $Q$ and $Q_{m}$ are of opposing signs are considered in $\S 7$. From the four cases presented above, it is clear that penetrative convection is possible in cases 2, 3, and 4. Hence it is these cases that are of interest in the following analysis. It is evident from the steady-state temperature profiles that the position of the turning point determines the stability characteristics of the system. To investigate what effect $Q$ and $Q_{m}$ have on the system, we assume that $d, d_{m}, \lambda, \lambda_{m}$ and $\epsilon_{T}$ are all fixed. Then $\hat{\epsilon} \propto 1 / Q, \hat{\epsilon}_{m} \propto 1 / Q_{m}$, and we consider what effect $\hat{\epsilon}$ and $\hat{\epsilon}_{m}$ have on the turning points $z_{t p}, z_{t p}^{m}$. 


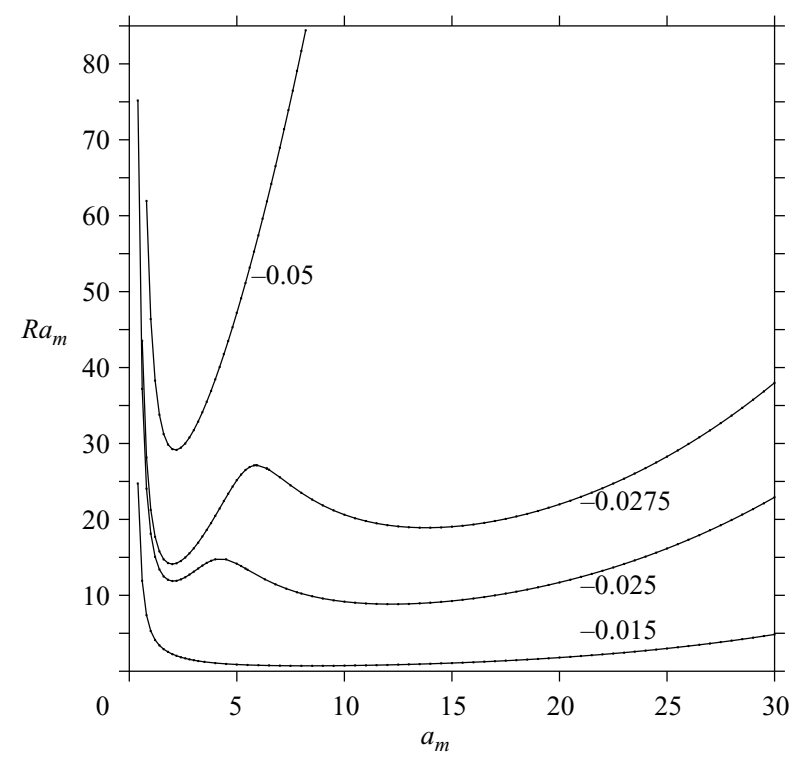

FIGURE 6. Neutral instability curves, $R a_{m}$ against $a_{m} . T_{U}=9{ }^{\circ} \mathrm{C}, \hat{d}=1, \hat{\epsilon}_{m}=-0.0728$, $\hat{\epsilon}$ varies as shown.

\subsection{Stability characteristics of $Q$}

Inspection of (6.4) reveals that as $\hat{\epsilon}$ becomes less negative, $z_{t p}$ increases for fixed $\hat{\epsilon}_{m}$. In other words, as the heat sink $Q$ becomes stronger, the system becomes more unstable. Similarly inspection of (6.5), shows as $\hat{\epsilon}$ becomes less negative, $z_{t p}^{m}$ increases for fixed $\hat{\epsilon}_{m}$. Once more, as $Q$ becomes stronger, the system becomes more unstable. Thus, $Q$ is destabilizing in cases 2,3 , and 4 , i.e. the stronger the heat sink in the fluid layer the more unstable the system. Physically this makes sense in all three cases.

Consider case 2: fluid at the interface is warmer and lighter than at the bottom of the porous medium. The lighter the fluid at the interface, the more stable the system. If the heat sink in the fluid is made stronger, the temperature at the interface will decrease and fluid there will become colder. Hence, the heat sink is destabilizing in this instance. In case 3, fluid at the interface is colder (and therefore heavier) than at the base of the porous medium. Therefore, if the heat sink in the fluid layer is made stronger, the temperature at the interface decreases and the difference between the fluid density at the interface and that at the base of the porous medium increases. Since the fluid at the interface is heavier than at the base, this is clearly destabilizing. In case 4, fluid at the interface is heavier than at the top of the fluid layer. Therefore, the whole of the fluid layer is potentially stable. However, if there is fluid within the layer that is colder than at the interface then there is a potentially unstable layer between the interface and the colder fluid. The stronger the heat sink in the fluid, the more likely this is (for fixed $Q_{m}$ ). Therefore, in this instance, a heat sink in the fluid is also destabilizing.

To be in keeping with Carr \& Straughan (2003), suppose the saturating fluid is water, and unless otherwise stated take $T_{L}=0{ }^{\circ} \mathrm{C}, \operatorname{Pr}=6, \epsilon_{T}=0.7, G_{m}=10, \alpha=0.1$ and $\delta=0.002$. In figure 6 the neutral curves for $T_{U}, \hat{d}$ and $\hat{\epsilon}_{m}$ fixed at $9{ }^{\circ} \mathrm{C}, 1$ and -0.0728 respectively, with $\hat{\epsilon}$ varying are presented. The neutral curves represent the variation of the porous Rayleigh number $R a_{m}$ with the porous wavenumber, $a_{m}$. The 
minimum value of $R a_{m}$ yields the critical value above which instability will commence. As $\hat{\epsilon}$ becomes less negative the neutral curves shift down and the system becomes more unstable. This is expected and in agreement with the analytical work presented above. $Q$ clearly has a destabilizing influence. Moreover, the neutral curves exhibit a bimodal nature like that first seen in Chen \& Chen (1988). For $\hat{\epsilon}=-0.05,-0.0275$ the minimum of the curve occurs at $a_{m} \approx 2$, whereas for $\hat{\epsilon}=-0.025,-0.015, a_{m} \approx 12,8.5$, respectively. Therefore as $\hat{\epsilon}$ becomes less negative, the shape of the convection cells switches from wide (smaller $a_{m}$ ) to narrow (larger $a_{m}$ ). If the critical wavenumber is small, the porous medium is said to dominate convection, whereas if it is large, the fluid layer dominates. The dominant layer refers to the layer in which instability commences. Thus, as $Q$ becomes stronger convection switches from porous to fluid initiated.

This is somewhat analogous to the findings of Worster (1992), who showed that in the solidification of binary alloys the onset of convection was dominated by one of two modes, mushy layer or boundary layer. The mushy-layer mode is driven by buoyant residual fluid within the mushy layer and induces a broad cellular motion in both the mushy layer and overlying fluid. The boundary-layer mode, on the other hand, is associated with a narrow compositional boundary layer in the melt and results in fine-scale convection in the boundary layer leaving the fluid in the mushy layer virtually stagnant. In the next section numerical findings are presented that exhibit a similar behaviour to those of Worster (1992), and yet are generated by a completely different physical process. In Worster (1992), the driving force behind convection is the release of latent heat and solute into the mushy layer, whereas here it is the removal of heat via sinks. The latent heat, first introduced by Worster (1986), is similar to the $Q_{m}$ term in the heat equation. Latent heat, however, is positive for solidification, whereas $Q_{m}$ is negative here. In $\S 7$ the case of $Q_{m}>0$ is considered. Worster (1992) does not report any penetrative effects. In the light of the work that follows, it is highly likely that for a given parameter range they do exist.

\subsection{Variation of the streamlines with $Q$}

In this section streamlines at criticality, corresponding to figure 6 are presented, i.e. $T_{U}, \hat{d}$ and $\hat{\epsilon}_{m}$ are fixed at $9^{\circ} \mathrm{C}, 1$ and -0.0728 respectively, and $\hat{\epsilon}$ is varied. Throughout all figures the porous-medium-fluid interface is at $z=0$, and the dashed line marks the turning point of the steady-state solution. Everywhere below the dashed line is unstably stratified while everywhere above is stably stratified.

When $\hat{\epsilon}=-0.0275$ (figure 7 ), there is one cell bridging the porous-medium-fluid interface and a further four cells are seen in the fluid above. The cell bridging the interface is the initiating cell. It is situated mainly below the unstable dashed line and thus drives convection. The four cells in the (stably stratified) fluid above are counter cells, a result of penetrative convection. Note that the centre of the driving cell is situated above the dashed line. This somewhat curious behaviour is a result of the resistance to movement the porous medium offers. This is explained in $\S 8$ where an example is given in which the driving cell is weaker than the counter cell above.

As the strength of $Q$ is increased (figure 8), the width of the cells becomes much narrower (the bimodal effect, cf. figure 6), and the depth of the unstable layer increases ( $Q$ is destabilizing). In this instance there are seven cells almost completely confined to the fluid layer with slight movement into the porous medium. An increase in the strength of $Q$ has clearly resulted in a switch in dominance, with the fluid layer playing a much stronger role in the convection pattern. 


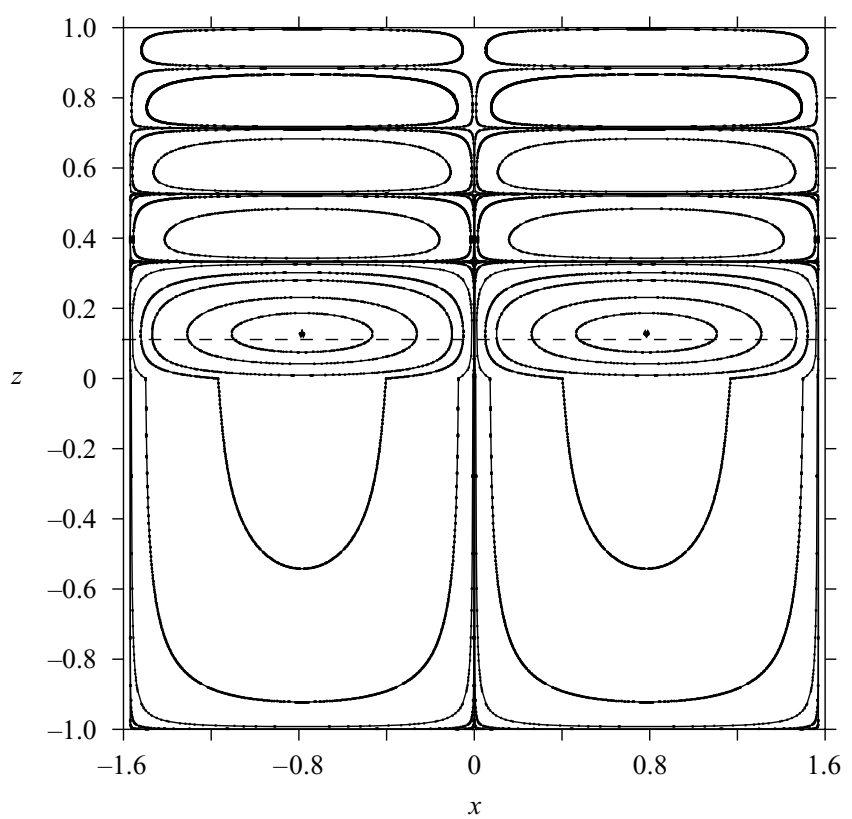

FIGURE 7. Streamline plot from the $Q$ model, type $4, T_{U}=9^{\circ} \mathrm{C}, \hat{d}=1, \delta=0.002$, $\hat{\epsilon}=-0.0275, \hat{\epsilon}_{m}=-0.0728 R a_{m}=14.112, a_{m}=2$.

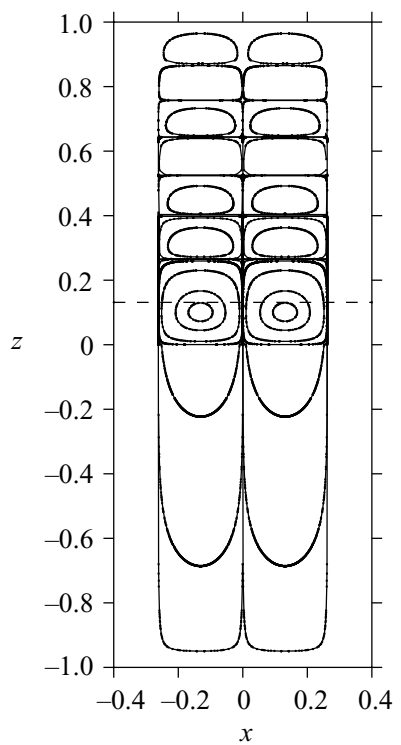

Figure 8. Streamline plot from the $Q$ model, type $4, T_{U}=9{ }^{\circ} \mathrm{C}, \hat{d}=1, \delta=0.002, \hat{\epsilon}=-0.025$, $\hat{\epsilon}_{m}=-0.0728 R a_{m}=8.839, a_{m}=12$.

\subsection{Stability characteristics of $Q_{m}$}

Inspection of (6.4), reveals that as $\hat{\epsilon}_{m}$ becomes less negative, $z_{t p}$ decreases for fixed $\hat{\epsilon}$. Therefore, when the turning point is in the fluid layer (case 4), the stronger $Q_{m}$ the more stable the system. In case 4, there is fluid within the fluid layer which is heavier than at the interface. Therefore, there is a potentially unstable layer between 


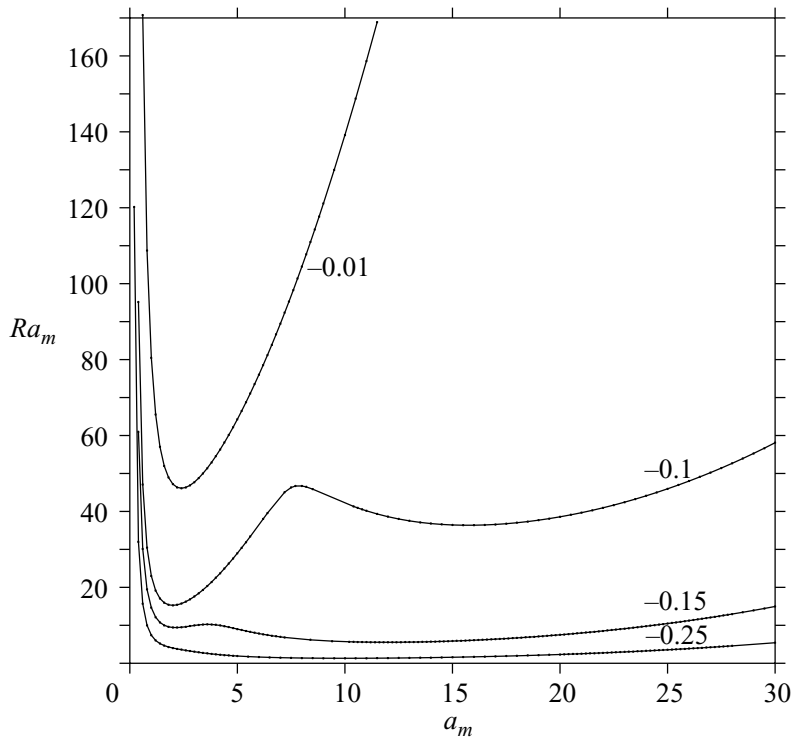

Figure 9. Neutral instability curves: $R a_{m}$ against $a_{m} . T_{U}=9{ }^{\circ} \mathrm{C}, \hat{d}=1, \hat{\epsilon}=-0.0357$, $\hat{\epsilon}_{m}$ varies as shown.

the interface and the colder fluid above. The stronger the heat sink in the porous medium, the colder the fluid at the interface (for fixed $Q$ ), and the smaller the density difference between fluid at the interface and the colder fluid above. Therefore, in this instance, the heat sink in the porous medium is stabilizing.

To investigate whether $Q_{m}$ is stabilizing or destabilizing when the turning point is in the porous layer is slightly more complicated. Inspection of (6.5) reveals that the effect of $\hat{\epsilon}_{m}$ depends on the sign of $\left(T_{U}-T_{L}+1 / \hat{\epsilon}\right)$. Suppose $\left(T_{U}-T_{L}+1 / \hat{\epsilon}\right)<0$, then as $\hat{\epsilon}_{m}$ becomes less negative, $z_{t p}^{m}$ decreases (for fixed $\hat{\epsilon}$ ). So in this case $Q_{m}$ is stabilizing. On the other hand suppose $\left(T_{U}-T_{L}+1 / \hat{\epsilon}\right)>0$, then as $\hat{\epsilon}_{m}$ becomes less negative, $z_{t p}^{m}$ increases. So in this instance $Q_{m}$ is destabilizing. Note that $\left(T_{U}-T_{L}+1 / \hat{\epsilon}\right)<0 \Rightarrow$ $T_{0}<T_{L}$, so $Q_{m}$ can only be stabilizing in case 3 such that $T_{U}-T_{L}<-1 / \hat{\epsilon}$. In all other instances for which the unstable layer is confined to the porous medium, i.e. case 2 and case 3 with $T_{U}-T_{L}>-1 / \hat{\epsilon}, Q_{m}$ is destabilizing.

To illustrate this curious behaviour more clearly consider figures 9-12. In figure 9, the neutral curves for $T_{U}, \hat{d}$ and $\hat{\epsilon}$ fixed at $9{ }^{\circ} \mathrm{C}, 1$ and -0.0357 respectively, with $\hat{\epsilon}_{m}$ varying are presented. As $\hat{\epsilon}_{m}$ becomes less negative the neutral curves shift up. Therefore, as $Q_{m}$ becomes stronger the system becomes more stable. This is in agreement with the analysis presented above since $T_{U}<-1 / \hat{\epsilon},\left(T_{L}=0{ }^{\circ} \mathrm{C}\right)$, so we expect an increase in the strength of $Q_{m}$ to have a stabilizing effect on the system. In figure 10 the corresponding steady-state temperature profiles are displayed. It is clear that as $\hat{\epsilon}_{m}$ becomes less negative the turning point moves down. Note that the steady-state temperature profiles are of type 3 and 4 only. Case 2 cannot be obtained since $T_{U}$ and $T_{L}$ violate (6.10).

In figure 11 , the neutral curves for $T_{U}, \hat{d}$ and $\hat{\epsilon}$ fixed at $15^{\circ} \mathrm{C}, 1$ and -0.1 respectively, with $\hat{\epsilon}_{m}$ varying are presented. In this instance, $\hat{\epsilon}_{m}$ has the opposite effect and $Q_{m}$ is clearly destabilizing. This is in agreement with the analysis presented above since $T_{U}>-1 / \hat{\epsilon}$. In figure 12 the corresponding steady-state temperature profiles are 


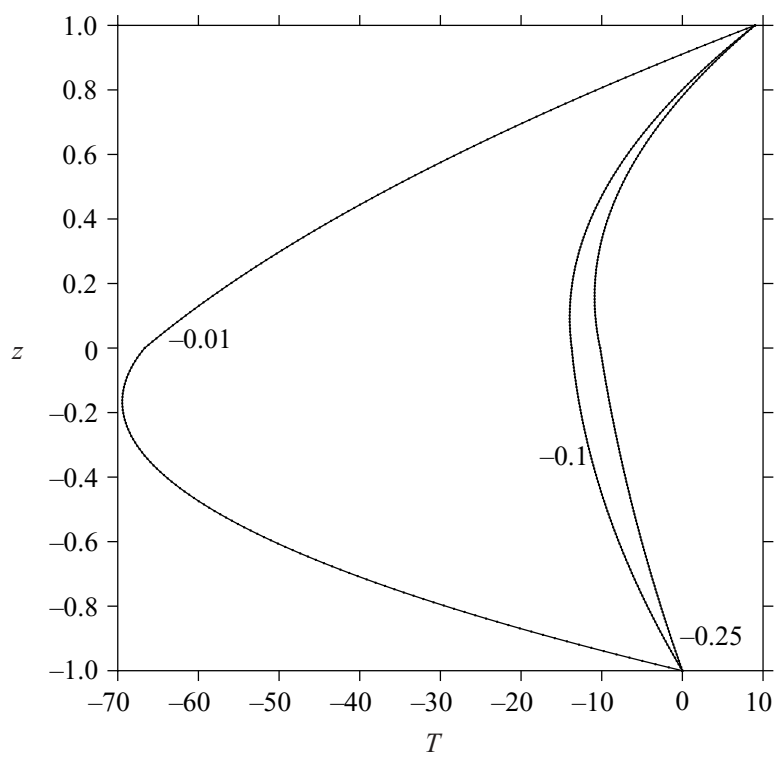

FigurE 10. Steady-state temperature profiles: depth $z$ against temperature $T . T_{U}=9{ }^{\circ} \mathrm{C}$, $\hat{d}=1, \hat{\epsilon}=-0.0357, \hat{\epsilon}_{m}$ varies as shown.

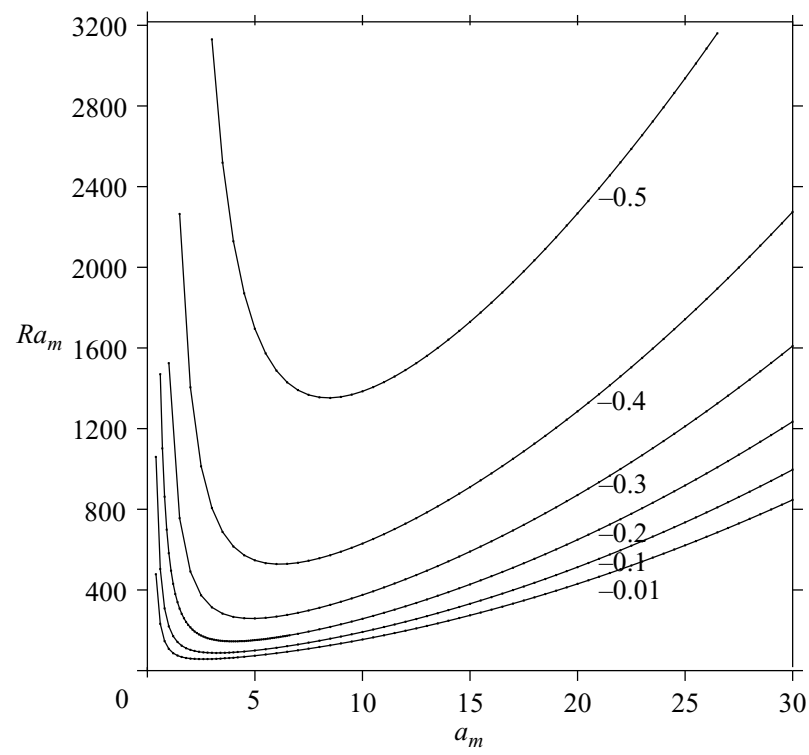

Figure 11. Neutral instability curves: $R a_{m}$ against $a_{m} . T_{U}=15^{\circ} \mathrm{C}, \hat{d}=1, \hat{\epsilon}=-0.1$, $\hat{\epsilon}_{m}$ varies as shown.

displayed. Note that they are of types 2 and 3 only. Case 4 cannot be obtained since $T_{U}$ and $T_{L}$ violate (6.6).

It has been shown analytically and in figures 11 and 12 that in case $2, Q_{m}$ is destabilizing. In case 2, there is fluid within the porous layer which is colder than at the base of the porous medium. This means there is a potentially unstable layer between the colder fluid and the base. As the heat sink in the porous medium is made 


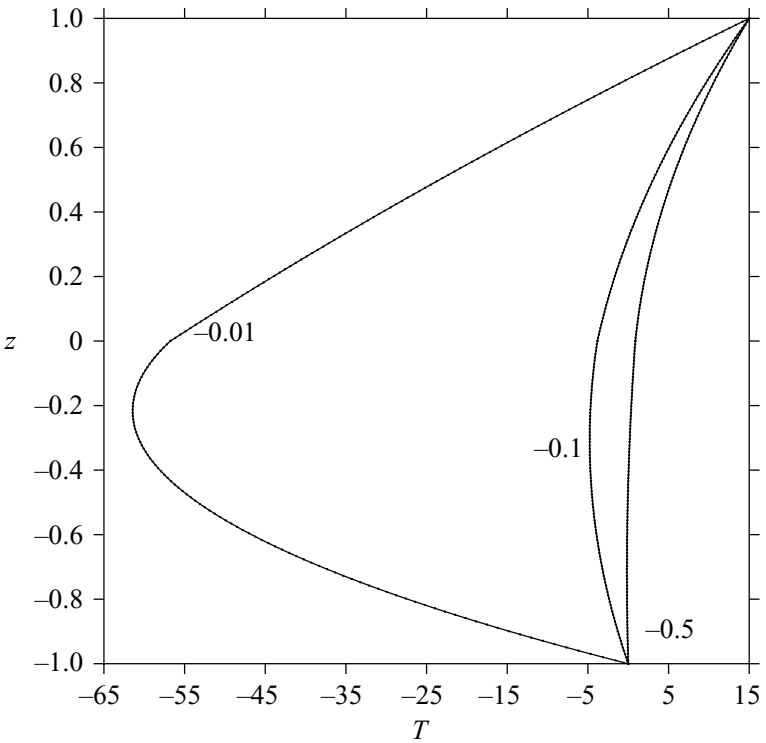

FIGURE 12. Steady-state temperature profiles: depth $z$ against temperature $T . T_{U}=15^{\circ} \mathrm{C}$, $\hat{d}=1, \hat{\epsilon}=-0.1, \hat{\epsilon}_{m}$ varies as shown.

stronger the temperature of the fluid within the porous medium decreases, while that at the base remains fixed. Hence as the heat sink in the porous medium becomes stronger the unstable layer becomes deeper (for fixed $Q$ ), and the heat sink in the porous medium has a destabilizing effect.

Explaining the effect of the porous heat sink in case 3 is not so straightforward. For fixed $T_{U}$ and $T_{L}$, it has been shown that the effect of the porous heat sink depends strongly upon the strength of the heat sink in the fluid. If the sink in the fluid is such that $\hat{\epsilon}>-1 /\left(T_{U}-T_{L}\right)$, then the sink in the porous medium is stabilizing. On the other hand, if $\hat{\epsilon}<-1 /\left(T_{U}-T_{L}\right)$ (weaker), then the sink in the porous medium is destabilizing. To clarify this, differentiate (6.5) with respect to $\hat{\epsilon}_{m}$ to yield

$$
\frac{\mathrm{d} z_{t p}^{m}}{\mathrm{~d} \hat{\epsilon}_{m}}=\frac{\mathscr{E} d_{m}}{2}\left(T_{U}-T_{L}+\frac{1}{\hat{\epsilon}}\right) .
$$

This implies that if $\hat{\epsilon}<-1 /\left(T_{U}-T_{L}\right)$ then $z_{t p}^{m}$ is an increasing function of $\hat{\epsilon}_{m}$. So as $\hat{\epsilon}_{m}$ increases, $z_{t p}^{m}$ increases and $Q_{m}$ is destabilizing. On the other hand, if $\hat{\epsilon}>-1 /\left(T_{U}-T_{L}\right)$ then $z_{t p}^{m}$ is a decreasing function of $\hat{\epsilon}_{m}$ and $Q_{m}$ is stabilizing. Clearly $\hat{\epsilon}=-1 /\left(T_{U}-T_{L}\right)$ is a local maximum and the critical point at which $Q_{m}$ switches from one behaviour to another. The strong interplay between the two heat sinks has a profound effect on the stability characteristics of $Q_{m}$ and a straightforward physical explanation is unclear.

In summary, the stability characteristics of $Q_{m}$ depend strongly on the steady-state temperature profile and the strength of $Q$. Figures 9 and 11 both show that as $Q_{m}$ becomes stronger the width of the convection cell changes from narrow $\left(a_{m}\right.$ larger) to wide $\left(a_{m}\right.$ smaller). Therefore, like $Q, Q_{m}$ has a dramatic effect on both the onset and nature of instability. This is illustrated more clearly in the next section, where a selection of streamlines corresponding to figures 9 and 11 at criticality are presented.

It is worth noting that in an entirely analogous analysis the effect of varying $\hat{d}$ and $T_{U}$ can also be investigated. Inspection of (6.4) and (6.5) implies that $\hat{d}$ and $T_{U}$ are 


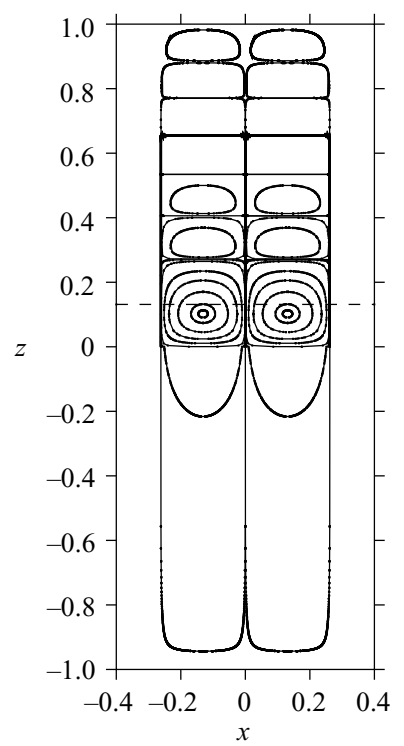

Figure 13. Streamline plot from the $Q$ model, type $4, T_{U}=9^{\circ} \mathrm{C}, \hat{d}=1, \delta=0.002$, $\hat{\epsilon}=-0.0357, \hat{\epsilon}_{m}=-0.15 R a_{m}=5.534, a_{m}=12$.

destabilizing and stabilizing parameters respectively. This is in agreement with the findings of Carr \& Straughan (2003).

\subsection{Variation of the streamlines with $Q_{m}$}

In figures 13 and $14, T_{U}, \hat{d}$ and $\hat{\epsilon}$ are fixed at 9,1 and -0.0357 respectively, and $\hat{\epsilon}_{m}$ is varied (cf. figure 9). When $\hat{\epsilon}_{m}=-0.15$ (figure 13), there are seven convection cells almost completely confined to the fluid layer with little movement into the porous medium. If $Q_{m}$ is made stronger (figure 14) the convection pattern changes completely. The cells become wider (the bimodal effect cf. figure 9), the depth of the driving cell decreases $\left(Q_{m}\right.$ is stabilizing) and the porous medium dominates the convection pattern.

In figures 15 and 16 , we concentrate on the behaviour of the streamlines when $Q_{m}$ is destabilizing. In this instance $T_{U}, \hat{d}$ and $\hat{\epsilon}$ are fixed at 15,1 and -0.1 respectively, and $\hat{\epsilon}_{m}$ is varied (cf. figure 11). Note that in figure 15 penetrative convection is seen in the porous medium. This is the first time such behaviour has been seen in the two-layer system, and we highlight this novel result. The relatively shallow depth of the unstable layer explains the occurrence of penetrative convection in the porous medium. The driving cell in the lower region of the porous medium induces penetrative convection in the stable porous medium and fluid above. However, the counter cells are very weak in comparison to the driving cell. Inspection of the eigenfunction reveals that the strongest counter cell (the one directly above the driving cell) is approximately 200 times weaker than the driving cell. The counter cells above are even weaker again.

In figure $16 Q_{m}$ is stronger than in figure 15 . In this instance the convection pattern is entirely different to that previously illustrated. The cells are wider, the unstable layer deeper, the driving cell spans the porous-medium-fluid interface and five counter cells are observed. Note that there is no switch in dominance between the two mediums in this case (instability is initiated in the porous medium in both instances), but the 


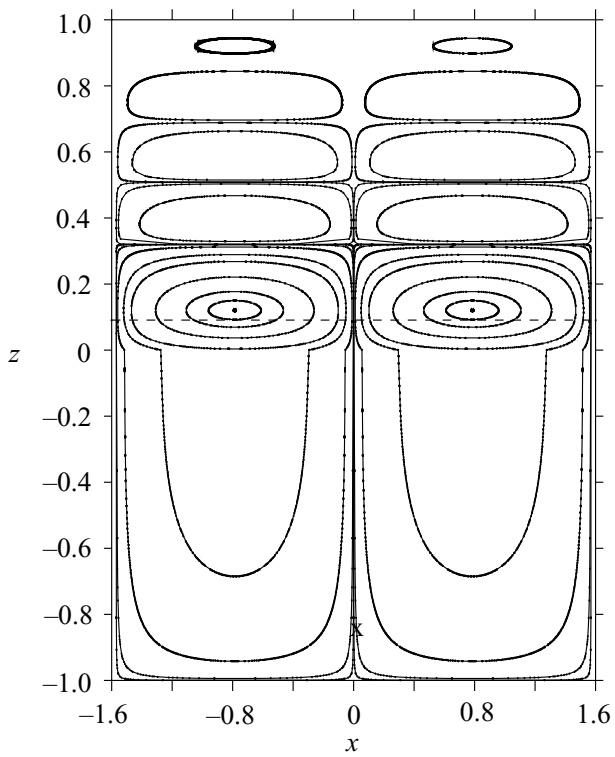

FIgURE 14. Streamline plot from the $Q$ model, type $4, T_{U}=9{ }^{\circ} \mathrm{C}, \hat{d}=1, \delta=0.002$, $\hat{\epsilon}=-0.0357, \hat{\epsilon}_{m}=-0.1 R a_{m}=15.262, a_{m}=2$.

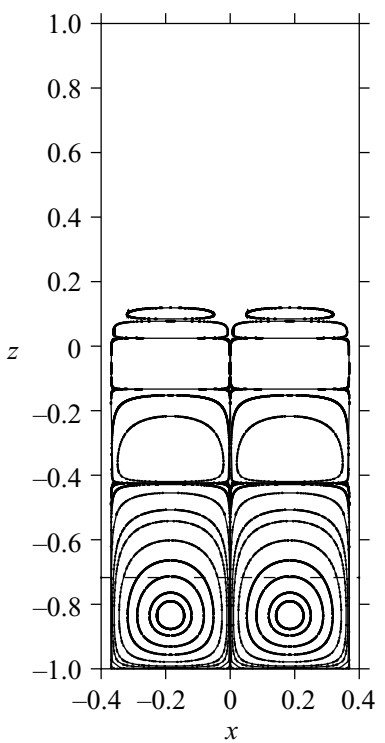

Figure 15. Streamline plot from the $Q$ model, type $2, T_{U}=15^{\circ} \mathrm{C}, \hat{d}=1, \delta=0.002$, $\hat{\epsilon}=-0.1, \hat{\epsilon}_{m}=-0.5 R a_{m}=1352.493, a_{m}=8.5$.

width of the cells does change from narrow to broad with an increase in $Q_{m}$ (cf. figure 11).

\section{General stability characteristics and discussion}

So far, attention has been restricted to the specific case of $Q, Q_{m}<0$. It is worth noting that $Q$ and $Q_{m}$ can be positive, negative or indeed zero by simple modification 


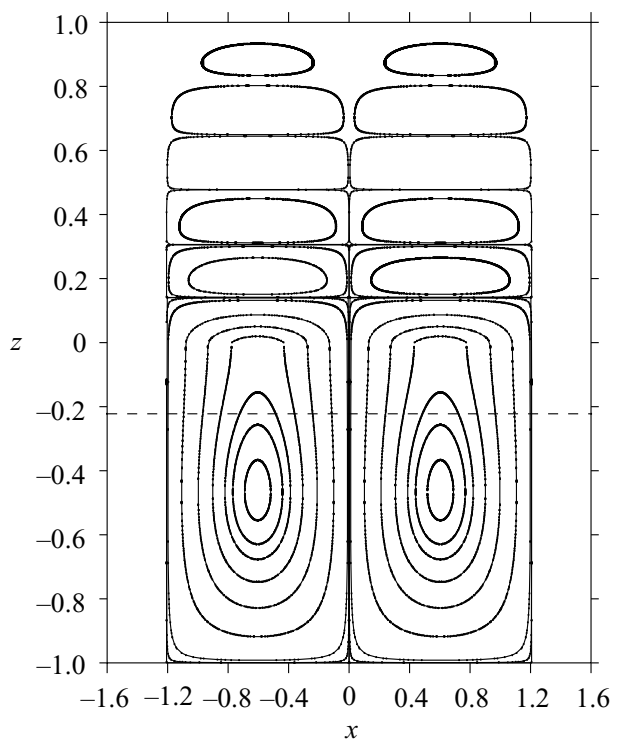

Figure 16. Streamline plot from the $Q$ model, type $3, T_{U}=15^{\circ} \mathrm{C}, \hat{d}=1, \delta=0.002$, $\hat{\epsilon}=-0.1, \hat{\epsilon}_{m}=-0.01 R a_{m}=57.061, a_{m}=2.6$.

\begin{tabular}{|c|c|c|c|c|c|}
\hline$Q$ & $Q_{m}$ & $\begin{array}{l}Q \text { effect } \\
\text { on fluid }\end{array}$ & $\begin{array}{c}Q \text { effect on } \\
\text { porous medium }\end{array}$ & $\begin{array}{l}Q_{m} \text { effect } \\
\text { on fluid }\end{array}$ & $\begin{array}{c}Q_{m} \text { effect on } \\
\text { porous medium }\end{array}$ \\
\hline Sink & Sink & D & D & S & $\begin{array}{l}\mathrm{D} \text { if } \gamma>-1 / \hat{\epsilon} \\
\mathrm{S} \text { if } \gamma<-1 / \hat{\epsilon}\end{array}$ \\
\hline Source & Source & $\begin{array}{l}\mathrm{S} \text { if } \gamma>1 / \hat{\epsilon}_{m} \\
\mathrm{D} \text { if } \gamma<1 / \hat{\epsilon}_{m}\end{array}$ & D & S & $\mathrm{S}$ \\
\hline Source & Sink & $\mathrm{S}$ & D & $\mathrm{S}$ & $\mathrm{D}$ \\
\hline Sink & Source & $\begin{array}{l}\mathrm{D} \text { if } \gamma>1 / \hat{\epsilon}_{m} \\
\mathrm{~S} \text { if } \gamma<1 / \hat{\epsilon}_{m}\end{array}$ & $\mathrm{D}$ & S & $\begin{array}{l}\mathrm{S} \text { if } \gamma>-1 / \hat{\epsilon} \\
\mathrm{D} \text { if } \gamma<-1 / \hat{\epsilon}\end{array}$ \\
\hline
\end{tabular}

TABLE 1 . Stability characteristics of $Q$ and $Q_{m}$, D denotes destabilizing and S stabilizing, $\gamma=T_{U}-T_{L}$.

of the non-dimensionalization and governing equations. This leads to a vast array of possible steady-state profiles and stability characteristics for $Q$ and $Q_{m}$. Table 1 provides a summary of the stability characteristics for all combinations of sources and sinks when $T_{U}>T_{L}$. Curiously, $Q$ has a destabilizing effect on the porous layer, in all cases, whereas $Q_{m}$ has a stabilizing effect on the fluid layer. On the other hand, the effect of $Q$ and $Q_{m}$ on their respective layers depends very much on the combination of sources and sinks under consideration, the strength of the source/sink in the opposing layer, and the temperature difference $T_{U}-T_{L}$.

Two very interesting steady states can be computed if $Q$ and $Q_{m}$ are of alternating sign. For example, figure 17 shows one possible state when $Q<0$ and $Q_{m}>0$. In this instance, an unstable layer bridging the interface is bounded above and below by stably stratified layers. Matthews (1988) considered a similar temperature profile when modelling the onset of penetrative convection in a layer of fluid. Matthews (1988) showed that convection in the unstable layer penetrated into the stably stratified 


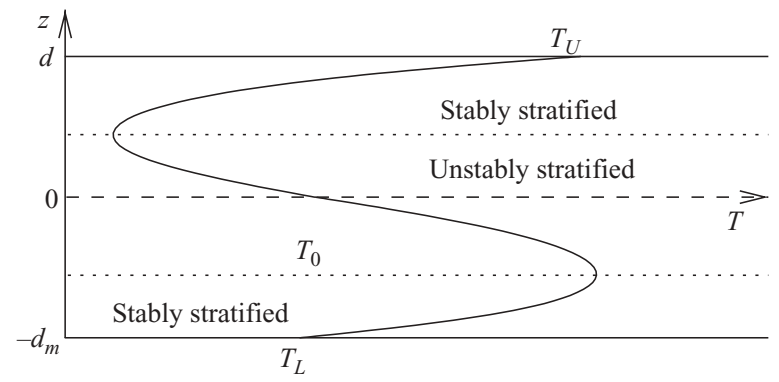

FIGURE 17. Steady-state temperature profile: depth $z$ against temperature $T$. $Q<0, Q_{m}>0$.

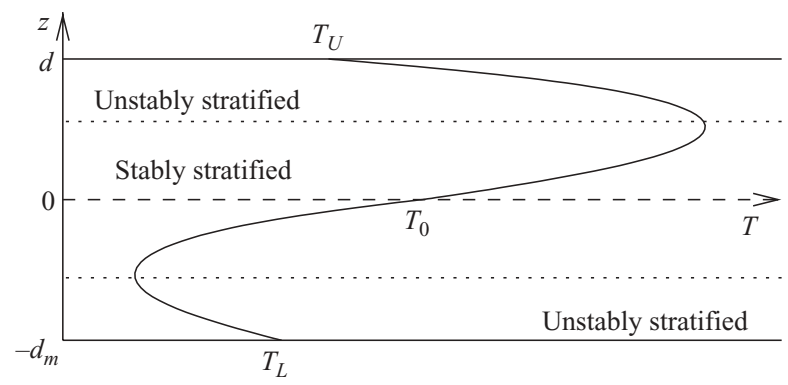

FIGURE 18. Steady-state temperature profile: depth $z$ against temperature $T . Q>0, Q_{m}<0$.

bounding layers. In the coupled porous-medium-fluid case a similar convection pattern occurs though there are natural differences. For example, if sources and sinks of equal strength are used in the two layers, the counter cell in the stably stratified porous layer is a lot weaker than its counterpart in the upper fluid layer (if it exists at all).

In figure 18, $Q>0$ and $Q_{m}<0$; in this case two unstably stratified layers bound a stable one from above and below. Normand \& Azouni (1992) considered a similar situation for a single layer of water when investigating penetrative convection near the density maximum. They found that the onset of convection could be via an oscillatory mode depending on the parameters governing the problem. Hence, resonant effects could occur in which convection oscillates between the unstable layers. Straughan (2004) investigated the porous analogue and found that the onset of instability was never oscillatory. It is not clear what effect coupling the two layers will have. Whether the coupled system will induce resonances (cf. Proctor \& Jones 1988), and how the coupling will affect the ensuing convection pattern is a very interesting problem but beyond the scope of the present paper.

\section{Comparison with a quadratic density model}

In Carr \& Straughan (2003) penetrative convection in the porous-medium-fluid system was modelled via a quadratic density profile. The governing equations here differ in that Carr \& Straughan (2003) do not include internal heating in either layer. Instead they model penetrative convection via a quadratic density profile (cf. Veronis 1963). Following an analysis similar to the one given in Tracey (1997), the model of Carr \& Straughan (2003), and the system presented in $\S 4$, can be written as $\boldsymbol{A}_{T^{2}} \boldsymbol{\Phi}=$ 
$\sigma \boldsymbol{B} \boldsymbol{\Phi}$ and $\boldsymbol{A}_{Q} \boldsymbol{\Phi}=\sigma \boldsymbol{B} \boldsymbol{\Phi}$ respectively, where $\boldsymbol{\Phi}=\left(\pi, u, v, w, \theta, \pi^{m}, u^{m}, v^{m}, w^{m}, \theta^{m}\right)^{T}$ and $u, u^{m}, v, v^{m}$ are the $\boldsymbol{i}, \boldsymbol{j}$-components of $\boldsymbol{u}$ and $\boldsymbol{u}^{m}$ respectively. The operators $\boldsymbol{A}_{T^{2}}$, $\boldsymbol{A}_{Q}$ and $\boldsymbol{B}$ are defined by

$$
\begin{aligned}
& \boldsymbol{A}_{T^{2}}=\left(\begin{array}{cccccccccc}
0 & -\frac{\partial}{\partial x} & -\frac{\partial}{\partial y} & -\frac{\partial}{\partial z} & 0 & \ldots & \ldots & \ldots & \ldots & 0 \\
-\frac{\partial}{\partial x} & \Delta & 0 & 0 & 0 & \ldots & \ldots & \ldots & \ldots & \vdots \\
-\frac{\partial}{\partial y} & 0 & \Delta & 0 & 0 & \ldots & \ldots & \ldots & \ldots & \vdots \\
-\frac{\partial}{\partial z} & 0 & 0 & \Delta & -2 R_{T^{2}}(\xi-z) & \ldots & \ldots & \ldots & \ldots & \vdots \\
0 & 0 & 0 & -R_{T^{2}} & \Delta & \ldots & \ldots & \ldots & \ldots & \vdots \\
0 & \ldots & \ldots & \ldots & \ldots & 0 & \delta^{2} \frac{\partial}{\partial x} & \delta^{2} \frac{\partial}{\partial y} & \delta^{2} \frac{\partial}{\partial z} & 0 \\
\vdots & \ldots & \ldots & \ldots & \ldots & \delta^{2} \frac{\partial}{\partial x} & 1 & 0 & 0 & 0 \\
\vdots & \ldots & \ldots & \ldots & \ldots & \delta^{2} \frac{\partial}{\partial y} & 0 & 1 & 0 & 0 \\
\vdots & \ldots & \ldots & \ldots & \ldots & \delta^{2} \frac{\partial}{\partial z} & 0 & 0 & 1 & 2 R_{T^{2}}^{m}\left(\xi_{m}-z\right) \\
0 & \ldots & \ldots & \ldots & \ldots & 0 & 0 & 0 & -R_{T^{2}}^{m} & \Delta
\end{array}\right), \\
& \boldsymbol{A}_{Q}=\left(\begin{array}{cccccccccc}
0 & -\frac{\partial}{\partial x} & -\frac{\partial}{\partial y} & -\frac{\partial}{\partial z} & 0 & \ldots & \ldots & \ldots & \ldots & 0 \\
-\frac{\partial}{\partial x} & \Delta & 0 & 0 & 0 & \ldots & \ldots & \ldots & \ldots & \vdots \\
-\frac{\partial}{\partial y} & 0 & \Delta & 0 & 0 & \ldots & \ldots & \ldots & \ldots & \vdots \\
-\frac{\partial}{\partial z} & 0 & 0 & \Delta & -R & 0 & \ldots & \ldots & \ldots & \vdots \\
0 & 0 & 0 & -R f(z) & \Delta & \ldots & \ldots & \ldots & \ldots & \vdots \\
0 & \ldots & \ldots & \ldots & \ldots & 0 & \delta^{2} \frac{\partial}{\partial x} & \delta^{2} \frac{\partial}{\partial y} & \delta^{2} \frac{\partial}{\partial z} & 0 \\
\vdots & \ldots & \ldots & \ldots & \ldots & \delta^{2} \frac{\partial}{\partial x} & 1 & 0 & 0 & 0 \\
\vdots & \ldots & \ldots & \ldots & \ldots & \delta^{2} \frac{\partial}{\partial y} & 0 & 1 & 0 & 0 \\
\vdots & \ldots & \ldots & \ldots & \ldots & \delta^{2} \frac{\partial}{\partial z} & 0 & 0 & 1 & -R^{m} \\
0 & \ldots & \ldots & \ldots & \ldots & 0 & 0 & 0 & R^{m} f^{m}(z) & \Delta
\end{array}\right)
\end{aligned}
$$

$$
\boldsymbol{B}=\left(\begin{array}{ccccccc}
0 & 0 & 0 & 0 & 0 & \ldots & 0 \\
0 & 1 & 0 & 0 & 0 & \ldots & 0 \\
0 & 0 & 1 & 0 & 0 & \ldots & 0 \\
0 & 0 & 0 & 1 & 0 & \ldots & 0 \\
0 & 0 & 0 & 0 & P r & \ldots & 0 \\
\vdots & & & & & \ddots & \vdots \\
0 & \ldots & & & & & \omega^{m}{ }^{m} G_{m}
\end{array}\right)
$$

where

$$
\begin{array}{ll}
R_{T^{2}}^{2}=\frac{g \bar{\alpha} d^{3} T_{U}^{2}(1-\mathscr{E})^{2}}{\nu \lambda}, & \xi=\frac{4 / T_{U}-\mathscr{E}}{1-\mathscr{E}} \\
R_{T^{2}}^{m 2}=\frac{g \bar{\alpha} K \operatorname{Pr}^{m} d_{m} T_{U}^{2} \mathscr{E}^{2}}{v^{2}}, & \xi_{m}=\frac{4 / T_{U}-\mathscr{E}}{\mathscr{E}}
\end{array}
$$




\begin{tabular}{crrrr}
$\hat{d}$ & \multicolumn{1}{c}{$R a_{T^{2}}^{m}$} & \multicolumn{1}{c}{$R a_{Q}^{m}$} & $a_{T^{2}}^{m}$ & $a_{Q}^{m}$ \\
0.05 & 273.311 & 273.322 & 5.0 & 5.0 \\
0.10 & 225.470 & 225.631 & 4.5 & 4.5 \\
0.50 & 61.942 & 62.844 & 2.5 & 2.5 \\
1.00 & 19.902 & 20.233 & 2.0 & 2.0
\end{tabular}

TABLE 2. The critical porous-medium Rayleigh number and wavenumber when the two models are adjoint.

Allowing $\boldsymbol{\Phi}_{1}$ and $\boldsymbol{\Phi}_{2}$ to be independent, it is not difficult to show that $\left\langle A_{T^{2}} \boldsymbol{\Phi}_{1}, \boldsymbol{\Phi}_{2}\right\rangle=$ $\left\langle\boldsymbol{\Phi}_{1}, A_{Q} \boldsymbol{\Phi}_{2}\right\rangle$ iff

$$
\begin{array}{cl}
R_{T^{2}}=R, & f(z)=2(\xi-z), \\
R_{T^{2}}^{m}=R^{m}, & f^{m}(z)=2\left(\xi_{m}-z\right) .
\end{array}
$$

Conditions (8.1) and (8.2) are satisfied iff

$$
\begin{gathered}
\hat{\epsilon}=\frac{-1}{T_{U}^{2}(1-\mathscr{E})^{2}}, \quad T_{0}=T_{U}+\frac{1-2 \xi}{\hat{\epsilon}}, \\
\hat{\epsilon}_{m}=\frac{-1}{T_{U}^{2} \mathscr{E}^{2}}, \quad T_{0}=T_{L}+\frac{2 \xi^{m}+1}{\hat{\epsilon}_{m}} .
\end{gathered}
$$

Substituting $(8.3 a)$ into $(8.3 b),(8.4 a)$ into $(8.4 b)$ and eliminating $T_{0}$ we find that

$$
T_{U}\left(9-T_{U}\right)=T_{L}
$$

Then, choosing $T_{L}=0{ }^{\circ} \mathrm{C}, T_{U}=9^{\circ} \mathrm{C}$ and $\hat{\epsilon}, \hat{\epsilon}_{m}$ such that (8.3a), (8.4a), are satisfied, we have $\boldsymbol{A}_{Q}^{*}=\boldsymbol{A}_{T^{2}}$ (where $\boldsymbol{A}_{Q}^{*}$ is the adjoint of $\boldsymbol{A}_{Q}$ ). In this case the two linearized models give rise to the same instability boundary, and we expect the two models to yield the same critical eigenvalues. In table 2, a comparison of the critical porous-medium Rayleigh numbers and wavenumbers obtained from the two models is made. Very good agreement between the two is seen.

While the eigenvalues are in good agreement, the corresponding eigenfunctions are not. For example, consider the $W$ eigenfunctions for the two models when $\hat{d}=1$, $T_{U}=9^{\circ} \mathrm{C}$ and $\hat{\epsilon}, \hat{\epsilon}_{m}$ satisfy $(8.3 a),(8.4 a)$, as illustrated in figure 19 . Note that in computing the eigenfunctions both $W$ and $W_{m}$ have been normalized. The strongest cell, therefore, is always positive regardless of its true circulation. Hence the true direction of circulation cannot be determined from the eigenfunction. However, it can be concluded that wherever a sign change occurs a counter cell exists. Figure 19 clearly shows that the eigenfunctions exhibit different behaviour. The main difference between the two is the position of the strongest cell. In the $Q$ model it is the first cell which is the strongest, whereas in the $T^{2}$ scenario it is the second. To illustrate this more clearly consider the corresponding streamlines given in figures 20 and 21 .

Figures 20 and 21 clearly show that the $T^{2}$ and $Q$ models generate different streamlines despite being mathematically adjoint and yielding near exact eigenvalues. This is due to the fact that the physical processes that drive convection in the two models are very different. In the $T^{2}$ model it is that density is a quadratic function of temperature, whereas in the $Q$ model it is the inclusion of the heat sinks. The two models represent very different physical systems and this must be understood in their application and interpretation at the outset. 


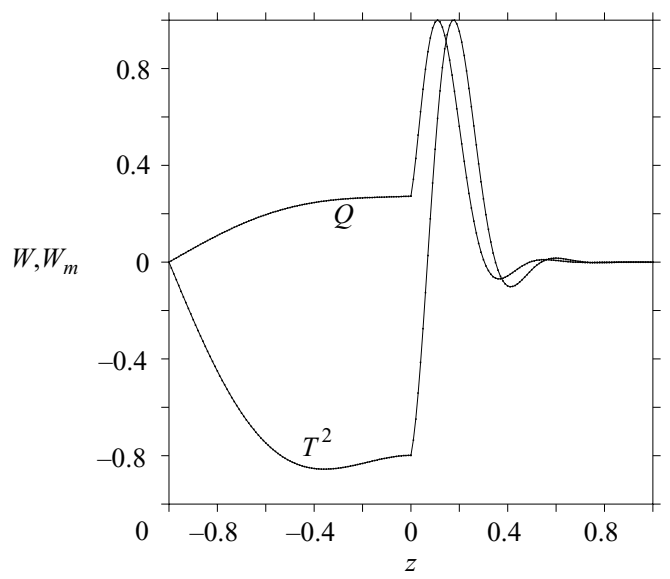

FigURE 19. $W$ eigenfunctions for the adjoint models, $T_{U}=9^{\circ} \mathrm{C} \hat{d}=1$.

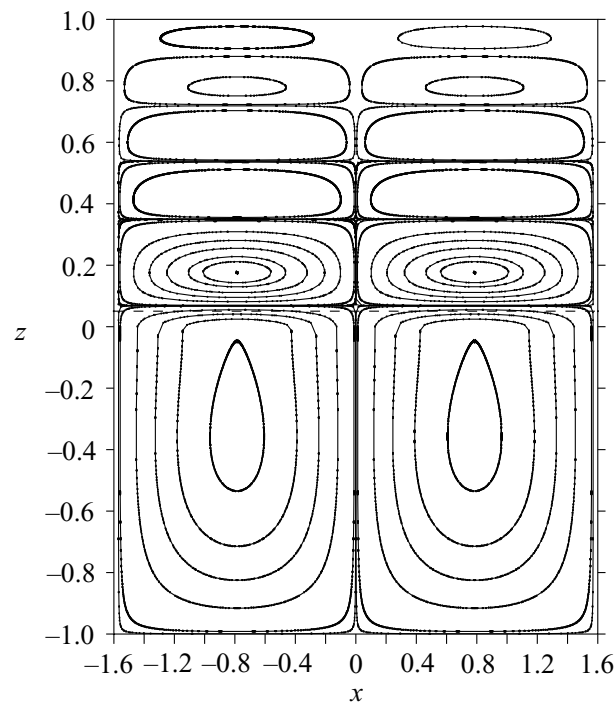

FIGURE 20. Streamline plot from the $T^{2}$ model, $T_{U}=9{ }^{\circ} \mathrm{C}, \hat{d}=1, \delta=0.002, R a_{m}=19.902$, $a_{m}=2$.

Figure 20 is curious in that the cell which initiates penetrative convection (the lower one confined mostly below the dashed line, where the dashed line marks the $4{ }^{\circ} \mathrm{C}$ conduction solution) is not as strong as the counter cell above. One explanation for this is the resistance to movement the porous medium offers. For example, if the porous medium were more permeable, the fluid within would be freer to move and we would expect the initiating cell to be stronger, cf. figure 22. Hence, care must be taken when interpreting data from the neutral instability curves. It is often inferred from the critical mode what the dominant layer is. As previously mentioned the dominant layer refers to the layer in which instability commences. However, this may not necessarily house the strongest convection cell.

The energy equation in both the $Q$ and $T^{2}$ models clearly shows that the Prandtl number, $P r$, is a parameter of the governing system. However, when $\operatorname{Pr}$ was varied 


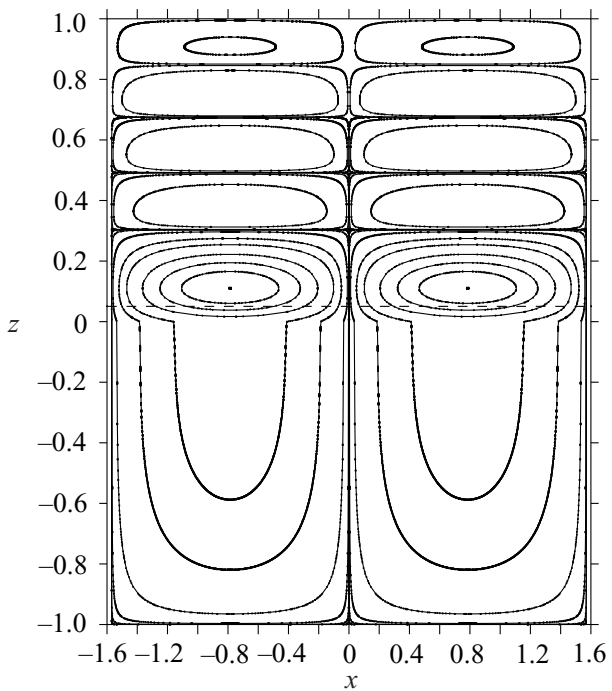

FIGURE 21. Streamline plot from the $Q$ model, type $4, T_{U}=9{ }^{\circ} \mathrm{C}, \hat{d}=1, \delta=0.002$, $\hat{\epsilon}=-0.0357, \hat{\epsilon}_{m}=-0.0728 R a_{m}=20.233, a_{m}=2$.

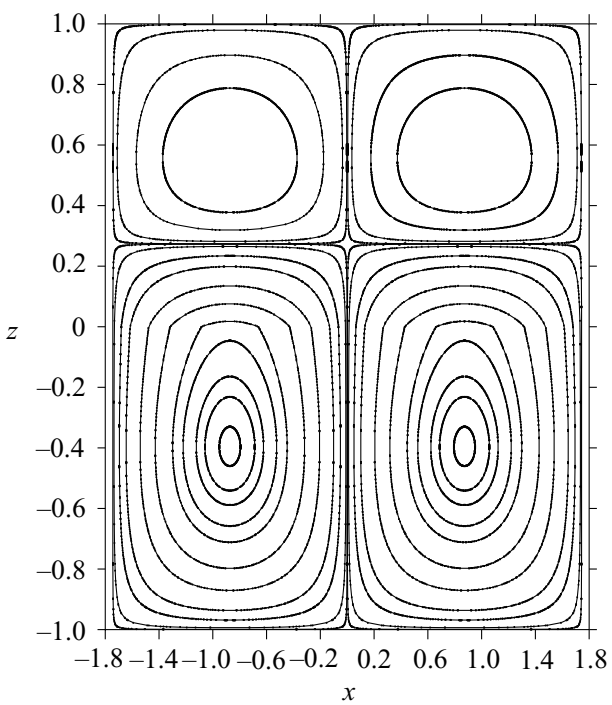

Figure 22. Streamline plot from the $T^{2}$ model, $T_{U}=9{ }^{\circ} \mathrm{C}, \hat{d}=1, \delta=0.1, R a_{m}=18.928$, $a_{m}=1.8$.

from 0.001 to 1000 , no significant difference was seen in the critical eigenvalues obtained from either model.

\section{Concluding remarks}

In this paper, penetrative convection for a two-layer system in which fluid overlies and saturates a porous medium has been modelled via internal heating. It was found that the instability of the system was very sensitive to change in the strength of the internal heating. It was shown analytically (and in some cases numerically) that $Q$ 
had a destabilizing effect on the porous layer, whereas $Q_{m}$ had a stabilizing effect on the fluid layer. The effects of $Q$ and $Q_{m}$ on their respective layers, however, depended upon the temperature difference $T_{U}-T_{L}$, and the strength of the heat source/sink in the opposing layer. An array of streamlines were presented to illustrate the effects of varying $Q$ and $Q_{m}$ on the pattern of instability.

The model presented was compared to Carr \& Straughan (2003). When mathematically adjoint, the two models were shown to yield the same critical instability boundaries but different eigenfunctions. Thus, the $Q$ model confirmed the instability boundaries of Carr \& Straughan (2003) and a need for caution and understanding when interpreting physical results was highlighted. Physically, it should be stressed that the $Q$ model is very different to the $T^{2}$ one.

The linear instability analysis provided valuable information about the nature and onset of instability. However, it did not preclude the possibility of subcritical instabilities and a nonlinear analysis is necessary to assess the validity of the linear work. A fully nonlinear analysis of the system presented here is highly non-trivial. It is our intent to develop a nonlinear stability analysis of the two-layer problem. It is hoped that the mathematical properties of the $Q$ model can be utilized in this task, cf. Straughan \& Walker (1996).

This work was supported by a research studentship of the Engineering and Physical Sciences Research Council. The author would like to thank Professor B. Straughan for his guidance, constructive criticism and discussion of the work. The author would also like to thank three anonymous referees for critical comments that have led to improvements in the paper.

\section{REFERENCES}

Allen, M. B. 1984 Collocation Techniques for Modeling Compositional Flows in Oil Reservoirs. Springer.

Allen, M. B. \& Khosravani, A. 1992 Solute transport via alternating-direction collocation using the modified method of characteristics. Adv. Water Resour. 15, 125-132.

Carr, M. \& Straughan, B. 2003 Penetrative convection in a fluid overlying a porous medium. Adv. Water Resour. 26, 262-276.

Chen, F. \& Chen, C. F. 1988 Onset of finger convection in a horizontal porous layer underlying a fluid layer. J. Heat Transfer 110, 403-409.

Chen, F. \& Chen, C. F. 1989 Experimental investigation of convective stability in a superposed fluid and porous layer when heated from below. J. Fluid Mech. 207, 311-321.

Chen, Q.-S., Prasad, V. \& Chatterjee, A. 1999 Modeling of fluid flow and heat transfer in a hydrothermal crystal growth system: Use of fluid-superposed porous layer theory. J. Heat Transfer 121, 1049-1058.

Curran, M. C. \& Allen, M. B. 1990 Parallel computing for solute transport models via alternating direction collocation. Adv. Water Resour. 13, 70-75.

Dongarra, J. J., Straughan, B. \& Walker, D. W. 1996 Chebyshev tau-QZ algorithm methods for calculating spectra of hydrodynamic stability problems. App. Numer. Maths 22, 399-434.

Eicken, H., Krouse, H. R., Kadko, D. \& Perovich D. K. 2002 Tracer studies of pathways and rates of meltwater transport through Arctic summer sea ice. J. Geophys. Res. 107, 22.1-22.20.

El-Habel, F., Mendoza, C. \& Bagtzoglou, A. C. 2002 Solute transport in open channel flows and porous streambeds. Adv. Water Resour. 25, 455-469.

EwING, R. E. 1996 Multidisciplinary interactions in energy and environmental engineering. J. Comput. Appl. Maths 74, 193-215.

Matthews, P. C. 1988 A model for the onset of penetrative convection. J. Fluid Mech. 188, 571-583.

NiELD, D. A. 1977 Onset of convection in a fluid layer overlying a layer of a porous medium. J. Fluid Mech. 81, 513-522. 
Nield, D. A. 1983 The boundary correction for the Rayleigh-Darcy problem: limitations of the Brinkman equation. J. Fluid Mech. 128, 37-46.

Nield, D. A. \& Bejan, A. 1999 Convection in Porous Media, 2nd Edn. Springer.

Normand, C. \& Azouni, A. 1992 Penetrative convection in an internally heated layer of water near the maximum density point. Phys. Fluids A 4, 243-253.

Notz, D., McPhee, M. G., Worster, M. G., Maykut, G. A., Schlunzen, K. H. \& Eicken, H. 2003 Impact of underwater-ice evolution on Arctic summer sea ice. J. Geophys. Res. 108, 16-28.

Proctor, M. R. E. \& Jones, C. A. 1988 The interaction of two spatially resonant patterns in thermal convection. Part 1. Exact 1:2 resonance. J. Fluid Mech. 188, 301-335.

RoberTs, P. H. 1967 Convection in horizontal layers with internal heat generation, Theory. J. Fluid Mech. 30, 33-49.

Straughan, B. 1993 Mathematical Aspects of Penetrative Convection. Longman.

StRAUGHAN, B. 2001 Effect of property variation and modelling on convection in a fluid overlying a porous layer. Intl J. Numer. Anal. Meth. Geomech. 26, 75-97.

Straughan, B. 2004 Resonant porous penetrative convection. Proc. R. Soc. Lond. A (in press).

Straughan, B. \& Walker, D. W. 1996 Anisotropic porous penetrative convection. Proc. R. Soc. Lond. A 452, 97-115.

Suchomel, B. J., Chen, B. M. \& Allen, M. B. 1998 a Network model of flow, transport and biofilm effects in porous media. Transport in Porous Media 30, 1-23.

Suchomel, B. J., Chen, B. M. \& Allen, M. B. $1998 b$ Macroscale properties of porous media from a network model of biofilm processes.Transport in Porous Media 31, 39-66.

TraceY, J. 1997 Stability analysis of multi-component convection-diffusion problems. PhD thesis, University of Glasgow.

Veronis, G. 1963 Penetrative convection. J. Astrophys. 137, 641-663.

Worster, M. G. 1986 Solidification of an alloy from a cooled boundary. J. Fluid Mech. 167, 481-501.

WORSTER, M. G. 1992 Instabilities of the liquid and mushy regions during solidification of alloys. $J$. Fluid Mech. 237, 649-669. 